\title{
New developments of the PANDA Disc DIRC detector
}

\author{
E. Etzelmüller4, R. Dzhygadlo ${ }^{1}$, A. Gerhardt ${ }^{1}$, K. Götzen ${ }^{1}$, R. Hohler ${ }^{1}$, G. Kalicy ${ }^{1}$, \\ H. Kumawat ${ }^{1}$, D. Lehmann ${ }^{1}$, B. Lewandowski ${ }^{1}$, M. Patsyuk ${ }^{1}$, K. Peters ${ }^{1}$, \\ G. Schepers ${ }^{1}$, L. Schmitt ${ }^{1}$, C. Schwarz ${ }^{1}$, J. Schwiening ${ }^{1}$, M. Traxler ${ }^{1}$, M. Zühlsdorf ${ }^{1}$, \\ V. Kh. Dodokhov ${ }^{2}$, A. Britting ${ }^{3}$, W. Eyrich ${ }^{3}$, A. Lehmann ${ }^{3}$, F. Uhlig ${ }^{3}$, M. Düren $^{4}$, \\ K. Föhl ${ }^{4}$, A. Hayrapetyan ${ }^{4}$, B. Kröck ${ }^{4}$, O. Merle ${ }^{4}$, J. Rieke ${ }^{4}$, E. Cowie ${ }^{5}$, T. Keri ${ }^{5}$, \\ R. Montgomery ${ }^{5}$, G. Rosner ${ }^{5}$, P. Achenbach ${ }^{6}$, M. Cardinali ${ }^{6}$, M. Hoek ${ }^{6}$, W. Lauth ${ }^{6}$, \\ C. Sfienti ${ }^{6}$, M. Thiel $^{6}$, P. Bühler ${ }^{7}$, L. Gruber ${ }^{7}$, J. Marton $^{7}$, K. Suzuki ${ }^{7}$
}

${ }^{1}$ GSI Helmholtzzentrum für Schwerionenforschung GmbH, Darmstadt, Germany

2 Joint Institute for Nuclear Research, Dubna, Russia

${ }^{3}$ Friedrich Alexander-University of Erlangen-Nuremberg, Erlangen, Germany

${ }^{4}$ II. Physikalisches Institut, Justus Liebig-University of Giessen, Giessen, Germany

${ }^{5}$ University of Glasgow, Glasgow, United Kingdom

${ }^{6}$ Institut für Kernphysik, Johannes Gutenberg-University of Mainz, Mainz, Germany

${ }^{7}$ Stefan Meyer Institut für subatomare Physik, Austrian Academy of Sciences, Vienna, Austria

E-mail: erik.etzelmueller@physik.uni-giessen.de

\begin{abstract}
The DIRC principle (Detection of Internally Reflected Cherenkov light) allows a very compact approach for particle identification detectors. The PANDA detector at the future FAIR facility at GSI will use a Barrel-DIRC for the central region and a Disc DIRC for the forward angular region between $5^{\circ}$ and $22^{\circ}$. It will be the first time that a Disc DIRC is used in a high performance $4 \pi$ detector. To achieve this aim, different designs and technologies have been evaluated and tested. This article will focus on the mechanical design and integration of the Disc DIRC with respect to the PANDA environment.
\end{abstract}

Technology and Instrumentation in Particle Physics 2014,

2-6 June, 2014

Amsterdam, the Netherlands

\footnotetext{
*Speaker.
} 


\section{Introduction}

At PANDA a stored and cooled antiproton beam of $1.5-15 \mathrm{GeV} / \mathrm{c}$ will collide with a fixed proton or nuclear target which allows the production of new particles with various quantum numbers. Besides a precise tracking and calorimetry, excellent particle identification (PID) is required to fully utilize the detector's capabilities in or-

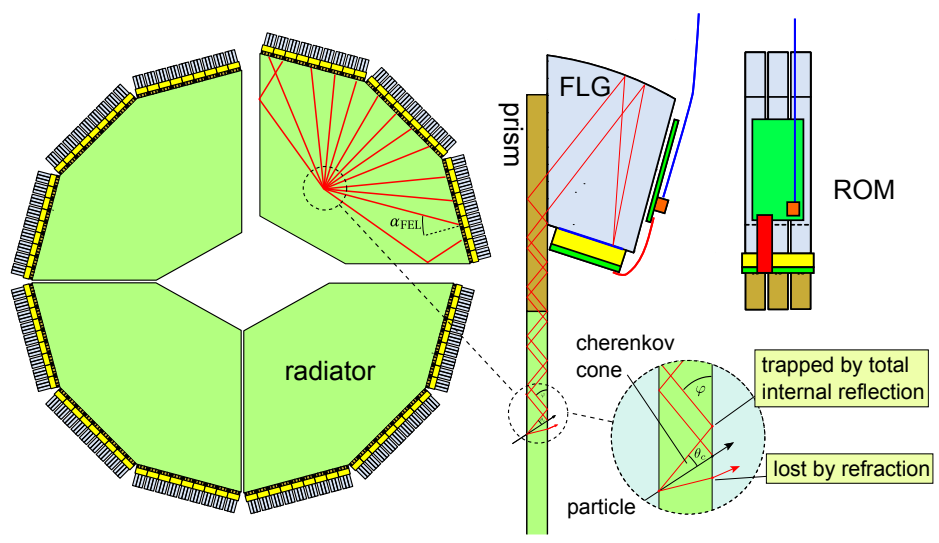

Figure 1: Working principle and design of the Disc DIRC [3] der to perform the envisaged physics program. Low momentum particles $(p<1 \mathrm{GeV} / \mathrm{c}$ ) will be identified using a time-of-flight detector, whereas higher momenta will be covered by RICH (Ring Imaging Cherenkov) detectors. In the very forward region $\left(\vartheta<5^{\circ}\right)$ a conventional RICH detector will be used. The remaining acceptance will be equipped with two detectors utilizing the DIRC (Detection of Internally Reflected Cherenkov light) principle. For angles between $22^{\circ}$ and $140^{\circ}$ a Barrel DIRC [1], which is based on the design of the BaBar DIRC [2], will enable $\pi / \mathrm{K}$-separation for momenta up to $3.5 \mathrm{GeV} / \mathrm{c}$. The remaining forward region will be equipped with a Disc DIRC which provides $\pi / \mathrm{K}$-separation for momenta up to $4 \mathrm{GeV} / \mathrm{c}$ [3].

Unlike a conventional RICH detector, a DIRC utilizes the radiator as a light-guide which makes it a very compact device. The radiator material therefore needs a higher refractive index which allows total internal reflection of the Cherenkov photons. The photons then propagate towards the side of the radiator where they can be detected [4].

\section{Design overview}

The design of the PANDA Disc DIRC is based on the concept of a focussing DIRC and is shown in figure 1 . Due to the large size of the acceptance area it was decided to divide the detector into four independent sub-detectors, called quadrants. Each quadrant consists of a large fused silica radiator which has the shape of a quarter of a regular dodecagon with a rhombic cutout at its center and a thickness of only $2 \mathrm{~cm}$. On the outer edges the radiator is extended by bars which guide the photons towards the FLGs (Focusing Light Guides). Both components are made of fused silica. The bars had to be introduced due to the varying spatial constraints for the FLGs and the requirement of having identical radiator plates. The FLGs have a cylindrical surface with an Al-coating, which focusses parallel entering photons on the focal plane, where they are detected by MCP-PMTs (Micro Channel Plate PhotoMultiplier Tubes) with a segmented anode $(3 \times 100$ pixels). The shape of the FLGs is optimized with respect to the magnetic field of the PANDA solenoid and the pitch of the MCP-PMT anode is adapted to the spot width of the focus. Three bars and FLGs share one MCP-PMT and form a ROM (ReadOut Module). 
The position information on the MCP-PMT anode in connection with the azimuthal position of the MCP-PMT provides a 2-dimensional information on the photon angle, which in connection with the momentum of the photon-emitting charged particle, gives a direct access to the Cherenkov angle $\theta_{c}$. The final track Cherenkov resolution is achieved by combining all photons per charged particle track. Timing information as a third dimension allows to separate background and resolve ambiguities [3].

Each ROM also contains the FEE (FrontEnd Electronics) which is currently planned to involve TOFPET ASICs [5] for the readout and the GBT chipset [6] for the data concentration.

\section{Mechanical design and integration}

Based on the final design, a mechanically feasible support integration had to be developed. As the PANDA Disc DIRC is the first of its kind, new ideas and concepts had to be evaluated.

The main challenges are the limited space and the secure and precise mounting of the optical components and sensors (see figures 2 and 3). The proposed design has the following key features:

- The full detector will be assembled on a mounting plate. The assembly can be done modularly and in the horizontal plane. After this is done, the mounting plate will be brought into a vertical position and screwed onto the holding structure of the subsequent electromagnet calorimeter.

- The optical system for each quadrant will form a rigid unit consisting of the radiator plate and 27 ROMs. The precise alignment can therefore be done prior to the installation and will not have to be repeated during operation.

- A thin stabilizing cross will be screwed tautly to the mounting plate. The cross will be made of thin aluminum profiles against which the individual quadrants can be pressed by spring-loaded connection blocks. This prevents the optical system from being shifted and receiving damage due to the different thermal expansion of the fused silica and the surrounding mechanics.

- The MCP-PMTs and FEEs are removable if needed. This is achieved by a mechanical holding structure which is glued to the optically non-critical surfaces of each ROM. The MCPPMTs will be pressed against the focal plane by spring-loaded

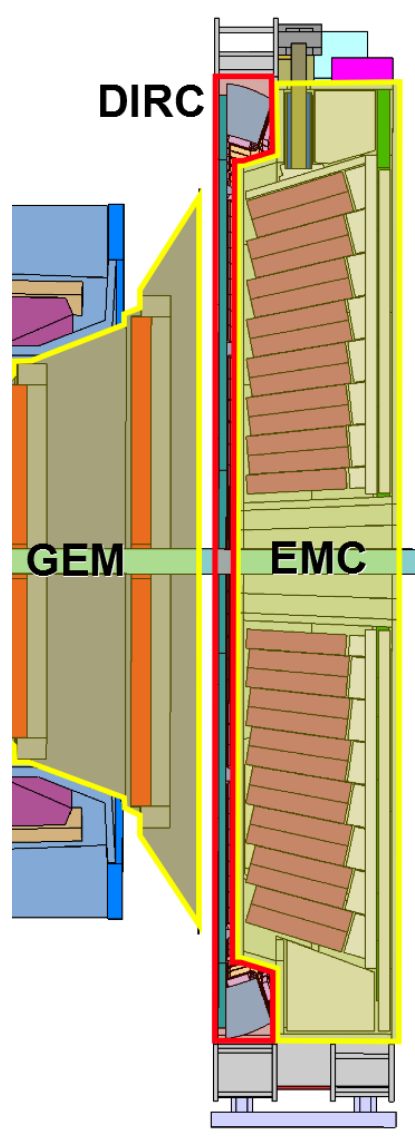

Figure 2: Sideview of the compact Disc DIRC in its dedicated environment. buttons.

In order to operate in a low moisture environment, the detector will be flushed with dry nitrogen gas similar to the BaBar DIRC [2]. In addition the whole detector will be put in gas- and light-tight covers. The cabling will be provided for each ROM individually. 

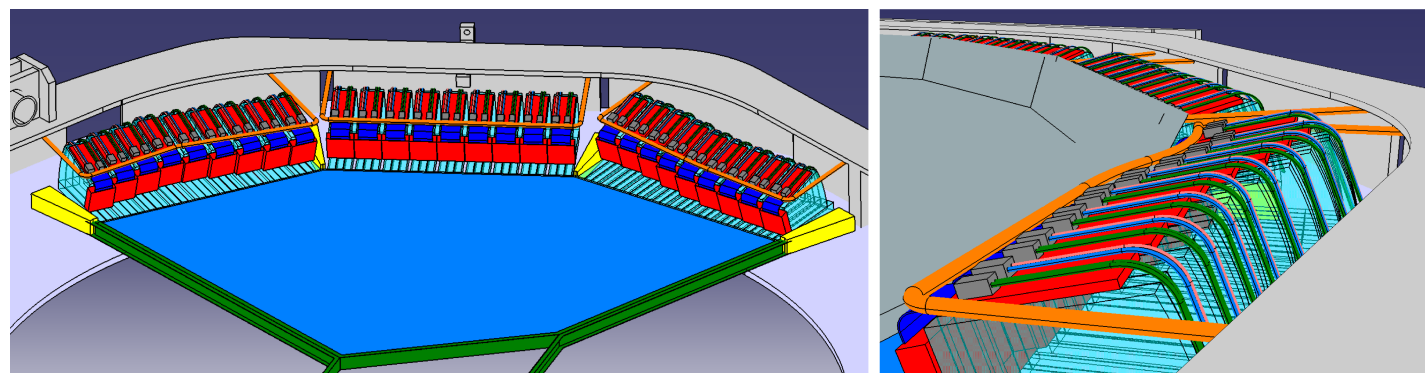

Figure 3: CAD pictures for the mechanical design. In the left illustration one can see the variation in the distance between the ROMs and the radiator. The right picture gives an impression of the tight spatial environment. Covers and ROM mechanics are not shown.

The assembly process is divided into four steps. The ROM assembly prepares the readout modules, afterwards the quadrant assembly consists of gluing the ROMs to the radiator and completing the setup of one quadrant which can afterwards be fully tested. While the former steps can be executed offsite, the final two steps need to be completed on-side in the experimental hall. During the horizontal assembly the quadrants are placed inside the stabilizing cross and all covers are sealed. The final step is the vertical assembly, at which the full detector is brought to its vertical position and mounted on the endcap's holding structure.

\section{Summary and outlook}

With the concepts for the mechanical design and integration at hand, one of the remaining challenges in order to realize a Disc DIRC detector has been overcome. Nevertheless all components have to be thoroughly tested regarding their functionality and durability. A mechanical mock-up of one quadrant with dimensions to scale is available for testing and will be especially useful regarding the cabling and assembly. The main focus, however, will be on the optical coupling and alignment between the radiator and the bars, as this glue line is crucial for a successful operation.

\section{References}

[1] M. Hoek et al., The PANDA Barrel DIRC detector, Nucl. Instr. Meth. A (2014) in press doi:http://dx.doi.org/10.1016/j.nima.2014.04.006

[2] I. Adam et al., The DIRC particle identification system for the BABAR experiment, Nucl. Instr. Meth. A 538 (2005) 281-357

[3] O. Merle et al., Development of an Endcap DIRC for PANDA, Nucl. Instr. Meth. A (2014) in press doi:http://dx.doi.org/10.1016/j.nima.2014.04.016

[4] T. Kamae et al., Focusing DIRC: A New compact Cherenkov ring imaging device, Nucl. Instr. Meth. A 382 (1996) 430-440

[5] M.D. Rolo et al., TOFPET ASIC for PET applications, JINST 8 (2013) C02050

[6] G. Mazza et al., A radiation tolerant $5 \mathrm{~Gb} / \mathrm{s}$ Laser Driver in $130 \mathrm{~nm}$ CMOS technology, JINST 7 (2012) C01052 